\title{
Incremental predictive value of the combined use of the neutrophil-to-lymphocyte ratio and systolic blood pressure difference after successful drug-eluting stent implantation
}

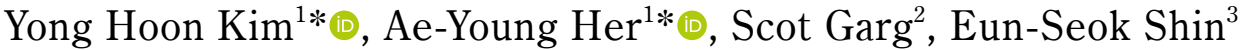 \\ ${ }^{1}$ Division of Cardiology, Department of Internal Medicine, Kangwon National University \\ School of Medicine, Chuncheon, Republic of Korea \\ ${ }^{2}$ East Lancashire Hospitals NHS Trust, Blackburn, Lancashire, United Kingdom \\ ${ }^{3}$ Division of Cardiology, Department of Internal Medicine, Ulsan University Hospital, Ulsan, Republic of Korea
}

\begin{abstract}
Background: Previous work has highlighted the importance of the neutrophil-to-lymphocyte ratio $(N L R)$ and the difference in the ward-to-catheterization laboratory systolic blood pressure $(\triangle S B P)$ in prognostic stratification after acute coronary syndrome. However, there is paucity of data regarding the added value of combining these two variables to predict 5-year major clinical outcomes after percutaneous coronary intervention.

Methods: A total of 1188 patients were classified into four groups according to the NLR and $\triangle S B P$ (high vs. low) using cutoffs derived from an analysis of receiver operating characteristic curves. A NLR $>3.0$ and $a \triangle S B P>25 \mathrm{mmHg}$ were considered high values. The primary endpoint was the composite of all-cause death, cardiac death, and non-fatal myocardial infarction. The secondary endpoint was the composite of target lesion revascularization, target vessel revascularization, and incidence of cerebrovascular accidents.

Results: The incidence of the primary endpoint was significantly higher in the high NLR and $\triangle S B P$ group than in the other three groups (2.2\% vs. $4.7 \%$ vs. $4.3 \%$ vs. $13.2 \%, p<0.001)$. The incidence of the secondary endpoint was similar among the four groups. Incorporation of high NLR and high $\triangle S B P$ into a model with conventional and meaningful clinical and procedural risk factors increased the $C$-statistics in predicting the primary endpoint ( 0.575 to $0.635, p=0.002$ ).

Conclusions: The power to predict the primary endpoint after drug-eluting stent implantation at the 5-year follow-up was improved by combining NLR and $\triangle S B P$. (Cardiol J 2023; 30, 1: 91-104)

Key words: blood pressure difference, drug-eluting stent, neutrophil-to-lymphocyte ratio, outcomes, percutaneous coronary intervention
\end{abstract}

\section{Introduction}

The fundamental mechanism of coronary artery disease (CAD) is stenosis caused by inflammation and atherosclerosis [1]. The earliest type of atherosclerotic lesion observed is a pure inflammatory lesion composed mainly of monocyte-derived macrophages and T-lymphocytes [2]. Arbel et al. [3] demonstrated that a high neutrophil-to-lymphocyte ratio (NLR) is significantly associated with higher

Address for correspondence: Eun-Seok Shin, MD, PhD, 877, Bangeojinsunhwando-ro, Dong-gu, Ulsan 44033, Republic of Korea, tel: +8252 250 7000, e-mail: sesim1989@gmail.com

Received: 1.03.2020 Accepted: $31.12 .2020 \quad$ Early publication date: 11.01.2021

*The first two authors (Yong Hoon Kim and Ae-Young Her) are equally contributed to this work.

This article is available in open access under Creative Common Attribution-Non-Commercial-No Derivatives 4.0 International (CC BY-NC-ND 4.0) license, allowing to download articles and share them with others as long as they credit the authors and the publisher, but without permission to change them in any way or use them commercially. 


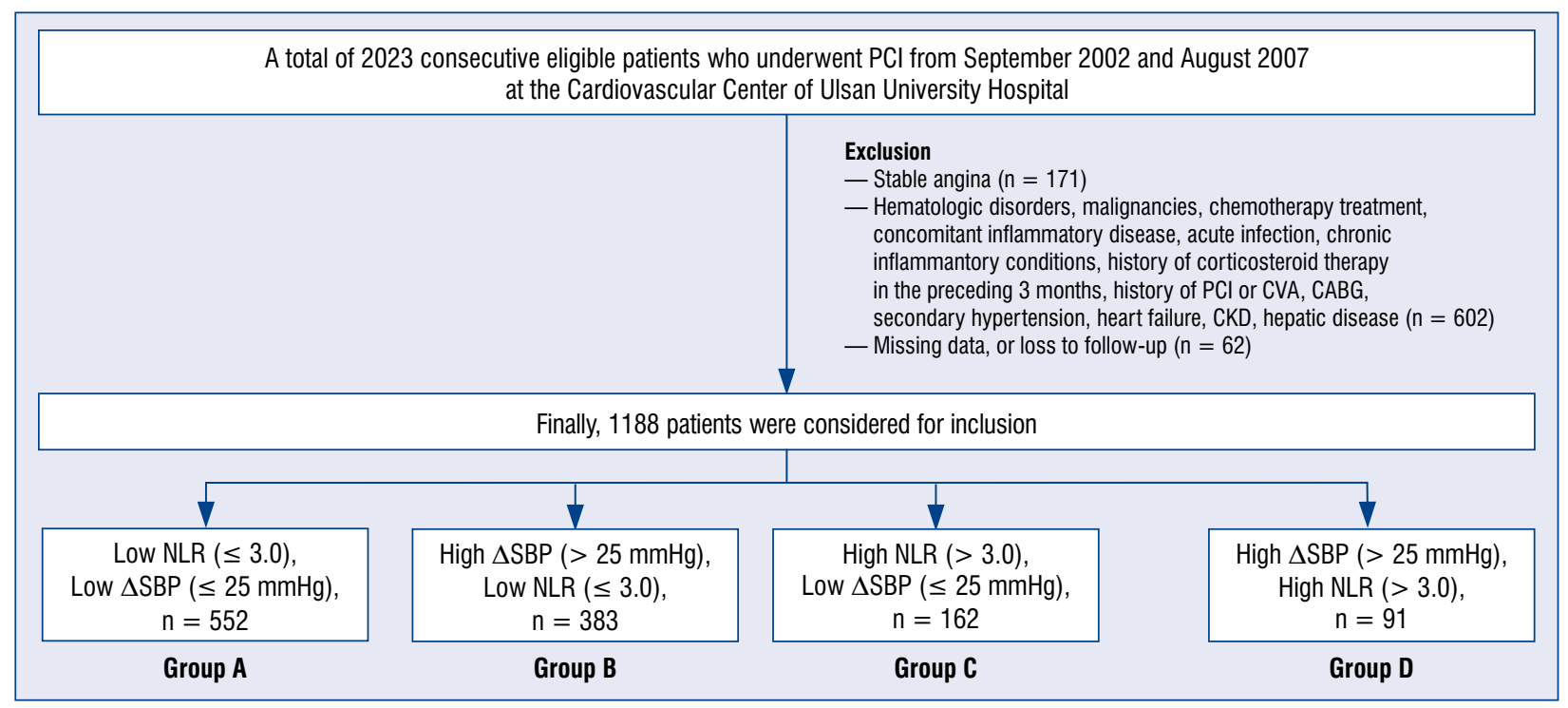

Figure 1. Flow chart; CABG — coronary artery bypass grafting; CKD — chronic kidney disease; CVA — cardiovascular accidents; NLR - neutrophil-to-lymphocyte ratio; PCl — percutaneous coronary intervention; $\Delta \mathrm{SBP}$ - differences in ward-to-catherization laboratory systolic blood pressure.

rates of 5-year mortality; therefore, the NLR could potentially be used to formulate prognosis in addition to conventional risk factors. Many other reports also emphasize the valuable role of NLR in $\mathrm{CAD}$ [4-6].

Another important causative factor of CAD is systolic arterial hypertension, which is associated with adverse cardiac events including such as death; as it is also associated with stress and other known psychosocial risk factors for CAD, it elevates the risk of cardiovascular sequelae [7, 8]. Coronary angiography (CAG) or percutaneous coronary intervention (PCI) may be accompanied by stress in healthy individuals. A meta-analysis suggested that greater responsiveness to acute mental stress has an adverse effect on future cardiovascular risk status; for example, a composite of elevated blood pressure (BP), increased left ventricular mass, subclinical atherosclerosis, and clinical cardiac events [9]. It would be very informative if we could predict long-term prognosis of the patients who are going to have CAG or PCI before these operations begin. Her et al. [10] suggested that changes in peri-procedural BP may be significantly associated with major adverse cardiac events and reported that a difference in the ward-to-catheterization laboratory systolic $\mathrm{BP}(\mathrm{SBP})(\triangle \mathrm{SBP})$ of $>20 \mathrm{mmHg}$ was related to an increased rate of all-cause death and cardiac death (CD) after drug-eluting stent (DES) implantation. In actual clinical practice, minimally invasive or non-invasive, inexpensive diagnostic tools are preferred over invasive diagnostic tools in view of cost and patient safety [11]. In this regard, the NLR and $\triangle \mathrm{SBP}$ are very useful non-invasive diagnostic tools for predicting adverse cardiac events. However, most previous studies $[4-6,10,12]$ focused only on one of those two parameters. Moreover, data showing the complementary actions and combined usefulness of NLR and $\triangle \mathrm{SBP}$ in patients diagnosed with acute coronary syndrome (ACS) are limited. Therefore, we investigated the additional predictive power of the NLR and $\triangle \mathrm{SBP}$ in comparison with that of conventional clinical and procedural risk factors in predicting 5 -year major clinical outcomes after DES implantation.

\section{Methods}

\section{Study design and population}

This retrospective observational study enrolled 2023 consecutive eligible patients who underwent PCI for ACS between September 2002 and August 2007 at the Cardiovascular Center of Ulsan University Hospital, Ulsan, South Korea. Data on cardiovascular risk factors and medical histories were self-reported by the patients. Patients were excluded if they had (1) stable angina $(\mathrm{n}=171$, 8.5\%); (2) any systemic diseases or treatment modality potentially affecting the white blood cells as shown in Figure 1 ( $\mathrm{n}=602,29.8 \%)$; and (3) missing data or patients were lost to follow-up $(\mathrm{n}=62,3.1 \%)$. Finally, 1188 patients were included in the study (Fig. 1). The study protocol complied 
with the ethical guidelines of the 1975 Declaration of Helsinki and was approved by the Ulsan University Hospital Institutional Review Board. Written informed consent was obtained from all participants. The enrolled patients were required to visit the cardiology out-patient department at the end of the first month and every 3 to 6 months thereafter for 5 years for clinical follow-up data to be collected through face-to-face interviews, medical chart reviews, and telephone contact.

\section{Study method and medical treatment}

During admission, venous blood samples were taken to assess the following: (1) complete blood cell counts, which included total white blood cells, differential counts (neutrophils, lymphocytes, monocytes, eosinophils), red blood cell, and platelet counts; (2) cardiac enzymes (creatine kinase myocardial band [CK-MB] and cardiac troponin T); and (3) other blood chemistry parameters (high-sensitivity C-reactive protein, serum creatinine, estimated glomerular filtration rate [eGFR], and lipid profiles). NLR was defined as the ratio of the neutrophil count to the lymphocyte count. The method of BP measurement has been described previously [10]. In brief, patients were measured with the resting right arm BP in the supine position in a ward setting, before transfer to the catheterization laboratory, where it was measured again after the patients had laid down on the catheterization laboratory table prior to any arterial puncture or PCI (ward-to-catheterization laboratory BP difference). Differences were estimated in the systolic and diastolic BP and heart rate obtained in the ward and catheterization laboratory, with the measurements taken by trained nurses using an auscultatory sphygmomanometer. The BP and heart rate were measured twice for each location, with at least a 1-minute interval between recordings and the mean values were used in the analysis. The stents were deployed using standard PCI techniques [13]. A successful PCI was defined as an angiographic residual stenosis diameter of $<30 \%$ in the presence of thrombolysis in myocardial infarction (TIMI) grade 3 flow. After DES implantation, a minimum of 1 year of dual antiplatelet therapy was administered (100 mg acetylsalicylic acid daily and $75 \mathrm{mg}$ clopidogrel daily).

\section{Study definitions and clinical endpoints}

The primary endpoint was the composite of allcause death, $\mathrm{CD}$, and non-fatal myocardial infarction (MI). The secondary endpoint was the composite of target lesion revascularization (TLR), target vessel revascularization (TVR), and cerebrovascular accidents at 5 -year follow-up. All-cause death was defined as either of CD or non-CD. Non-fatal MI was defined as the presence of clinical symptoms, electrocardiographic changes, or abnormal imaging findings of MI, combined with an increase in the CK-MB fraction above the upper normal limits or an increase in troponin-T/troponin-I to greater than the $99^{\text {th }}$ percentile of the upper normal limit after index PCI [14-16]. The definitions of TLR and TVR have been previously described [17]. The mean eGFR was calculated using the Modification of Diet in Renal Disease equation [18].

\section{Statistical analysis}

All statistical analyses were performed using SPSS v20 (IBM; Armonk, NY, USA). For continuous variables, differences among the three groups were evaluated using the analysis of variance or the Jonckheere-Terpstra test, and post-hoc analysis between the two groups was carried out using the Hochberg test or Dunnett-T3 test. Data are expressed as the means \pm standard deviations. For discrete variables, the differences between two out of the three groups were analyzed using the $\chi^{2}$ test or the Fisher exact test, as appropriate; data are expressed as counts and percentages [19]. In a multivariable Cox proportional hazard regression analysis, the baseline confounding covariates were selected if they were significantly different $(\mathrm{p}<0.001)$ among the four groups or between the two groups or had predictive values. The multivariable Cox proportional hazard regression analysis including baseline confounding factors, was used to compare the clinical endpoints among the four groups or between the two groups. Survival analysis among the four groups was performed using the Kaplan-Meier method, and differences between the two groups were assessed using the log-rank test. Receiver operating characteristic (ROC) curves were used to differentiate the ability of the NLR and $\triangle \mathrm{SBP}$ to predict primary endpoint (Fig. 2). After evaluating the relationship of the NLR and $\triangle \mathrm{SBP}$ with the clinical outcomes using Cox proportional hazard regression analysis, we compared the incremental value of combining a high NLR and a high $\triangle \mathrm{SBP}$ into the context of conventional and meaningful clinical and procedural characteristics for prediction of the primary endpoint. Estimates of the C-statistics for the Cox regression models were computed using the method of Pencina and D'Agostino [20]. Differences in the C-statistics (with 95\% confidence interval [CI]) 


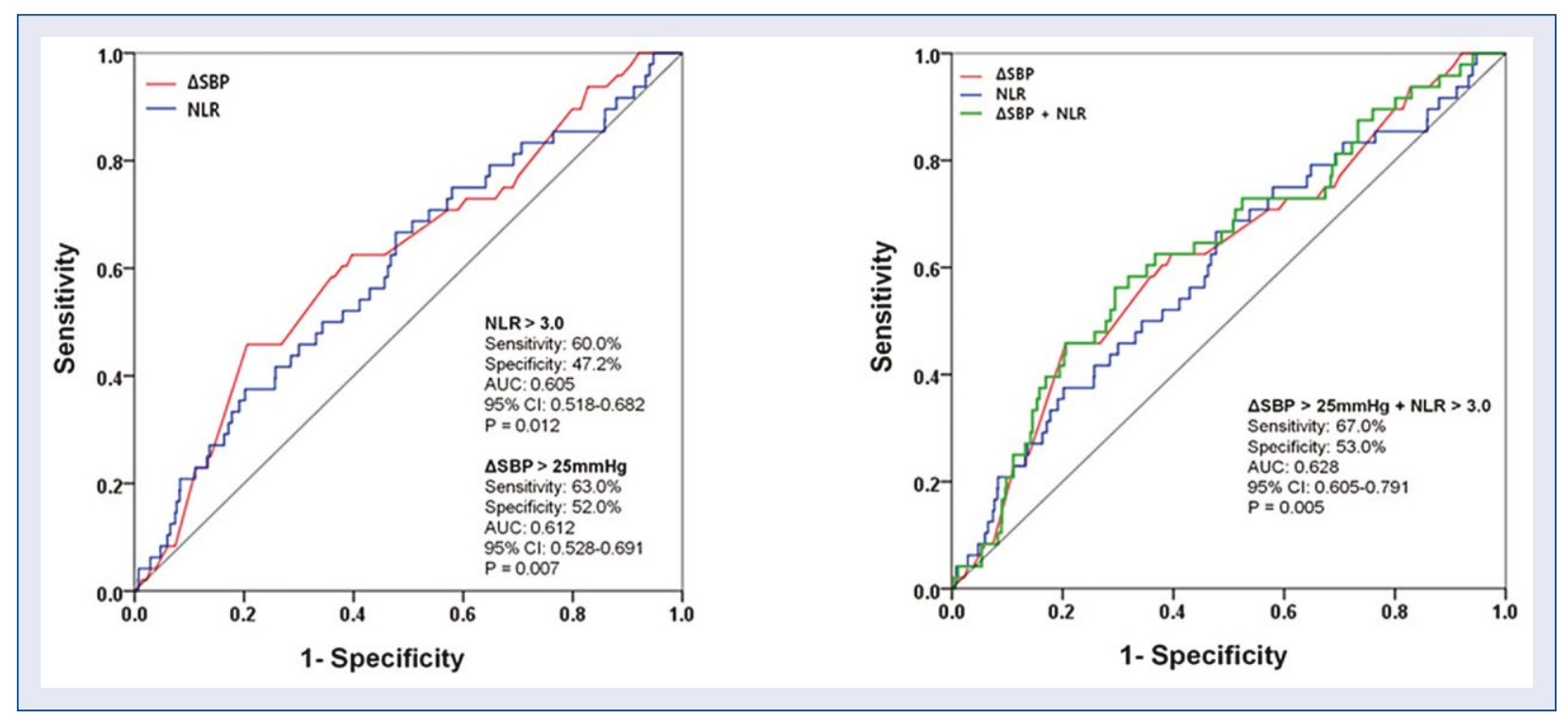

Figure 2. Receiver operating characteristic curve of neutrophil-to-lymphocyte ratio (NLR) and difference in the ward-to-catheterization laboratory systolic blood pressure $(\triangle \mathrm{SBP})$ for primary endpoints.

after the addition of the high NLR and high $\triangle \mathrm{SBP}$ to a model with conventional and meaningful clinical and procedural risk factors were obtained using the bootstrap percentile method (200 replicates) [21]. The statistical significance level was set at a p-value of $<0.05$ using a two-tailed test.

\section{Results}

\section{Cutoff values for the NLR and $\triangle \mathrm{SBP}$}

Analysis of the ROC curve was performed to detect the best NLR cutoff value that predicted the primary endpoint. This yielded a cutoff NLR of 3.0 , with a sensitivity of $60.0 \%$, a specificity of $47.2 \%$, and an area under the ROC curve of 0.605 (95\% CI: $0.518-0.682$ ) (Fig. 2). Therefore, NLRs of $>3.0(\mathrm{n}=253,21.3 \%)$ and $\leq 3.0$ $(\mathrm{n}=935,78.7 \%)$ were considered to be high and low values, respectively. Thereafter, the subjects were sub-divided according to $\triangle \mathrm{SBP}$. In the same manner, an ROC curve analysis was used to detect the best cutoff value of $\Delta \mathrm{SBP}$ for predicting the primary endpoint. This yielded a cutoff $\Delta \mathrm{SBP}$ of $25 \mathrm{mmHg}$, with a sensitivity of $63.0 \%$, a specificity of $52.0 \%$, and an area under the ROC curve of 0.612 (95\% CI: $0.528-0.691$; Fig. 2 ). $\triangle \mathrm{SBP}$ $>25 \mathrm{mmHg}(\mathrm{n}=474,39.9 \%)$ and $\leq 25 \mathrm{mmHg}$ ( $\mathrm{n}=714,60.1 \%)$ were considered to be high and low values, respectively. The patients were classified into four groups according to the NLR and $\triangle \mathrm{SBP}$ (high vs. low) using the cutoffs derived from the analysis of the ROC curves: group A (low NLR $[\leq 3.0]$ and low $\triangle \mathrm{SBP}[\leq 25 \mathrm{mmHg}], \mathrm{n}=552$, $46.5 \%$ ), group $\mathrm{B}$ (high $\Delta \mathrm{SBP}[>25 \mathrm{mmHg}]$ and low NLR $[\leq 3.0], \mathrm{n}=383,32.2 \%$ ), group $\mathrm{C}$ (high NLR [ $>3.0]$ and low $\Delta \mathrm{SBP}[\leq 25 \mathrm{mmHg}], \mathrm{n}=162$, $13.6 \%$ ), and group $\mathrm{D}$ (high $\Delta \mathrm{SBP}[>25 \mathrm{mmHg}]$ and high NLR [>3.0], $\mathrm{n}=91,7.7 \%)$.

\section{Baseline clinical and angiographic characteristics}

The baseline laboratory and angiographic characteristics according to the NLR and $\triangle$ SBP are summarized in Table 1 . The mean age of the total study population was $60.5 \pm 10.3$ years, and the oldest patient was included in group D. The mean left ventricular ejection fraction (LVEF) of the total study population was $61.6 \pm 10.3 \%$. The number of patients with hypertension and unstable angina was the highest in group $\mathrm{B}$. The number of acute $\mathrm{MI}$ was the highest in group $\mathrm{C}$. The mean serum creatinine level was the highest in group $\mathrm{D}$. However, the number of patients with a history of diabetes mellitus, dyslipidemia, and MI; number with left anterior descending artery, left circumflex artery, and right coronary artery as the treated vessels; American College of Cardiology/ /American Heart Association lesion type; extent of CAD; number of deployed stents; mean diameter of deployed stents; and mean length of deployed stents were not significantly different among the four groups. 


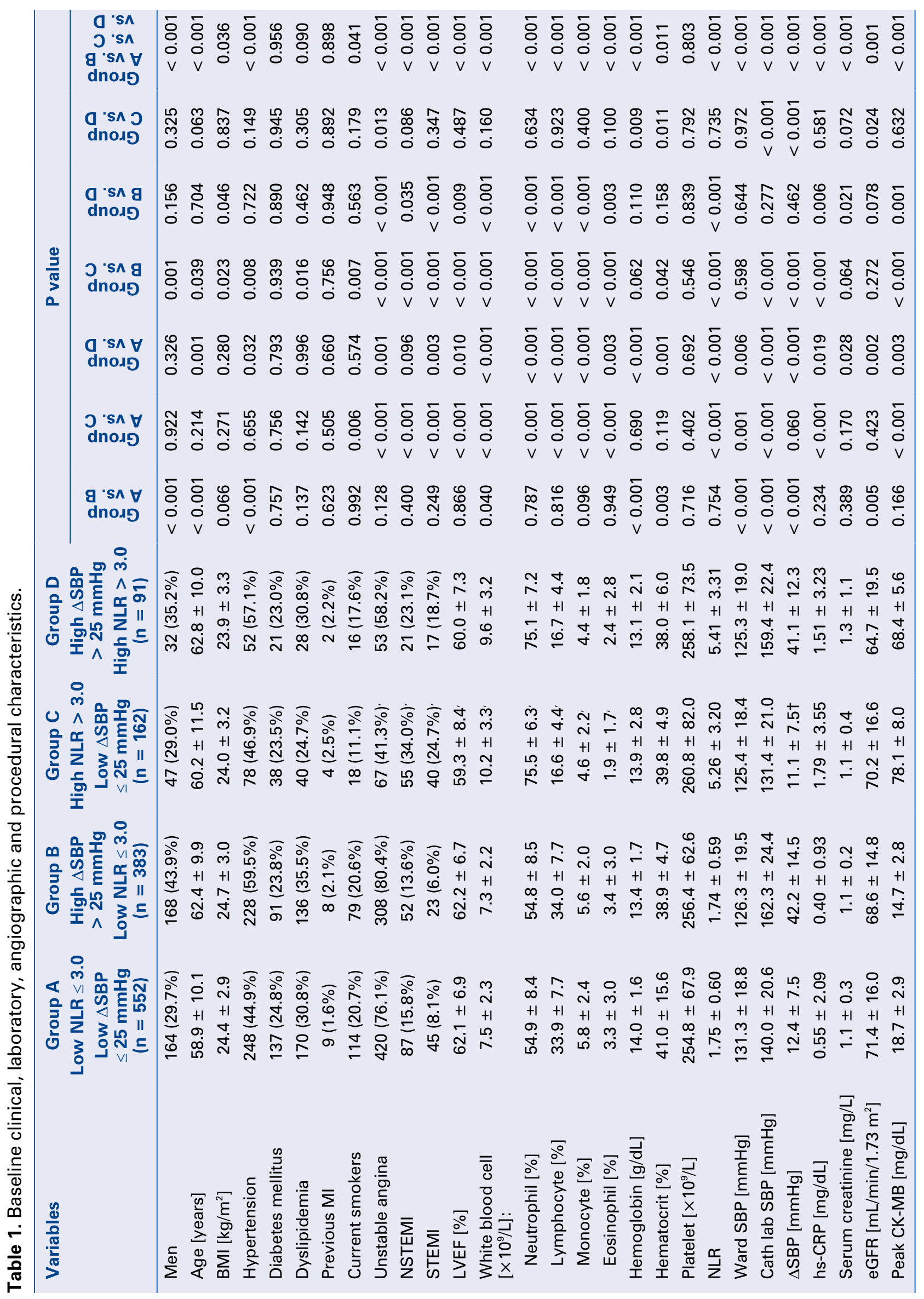




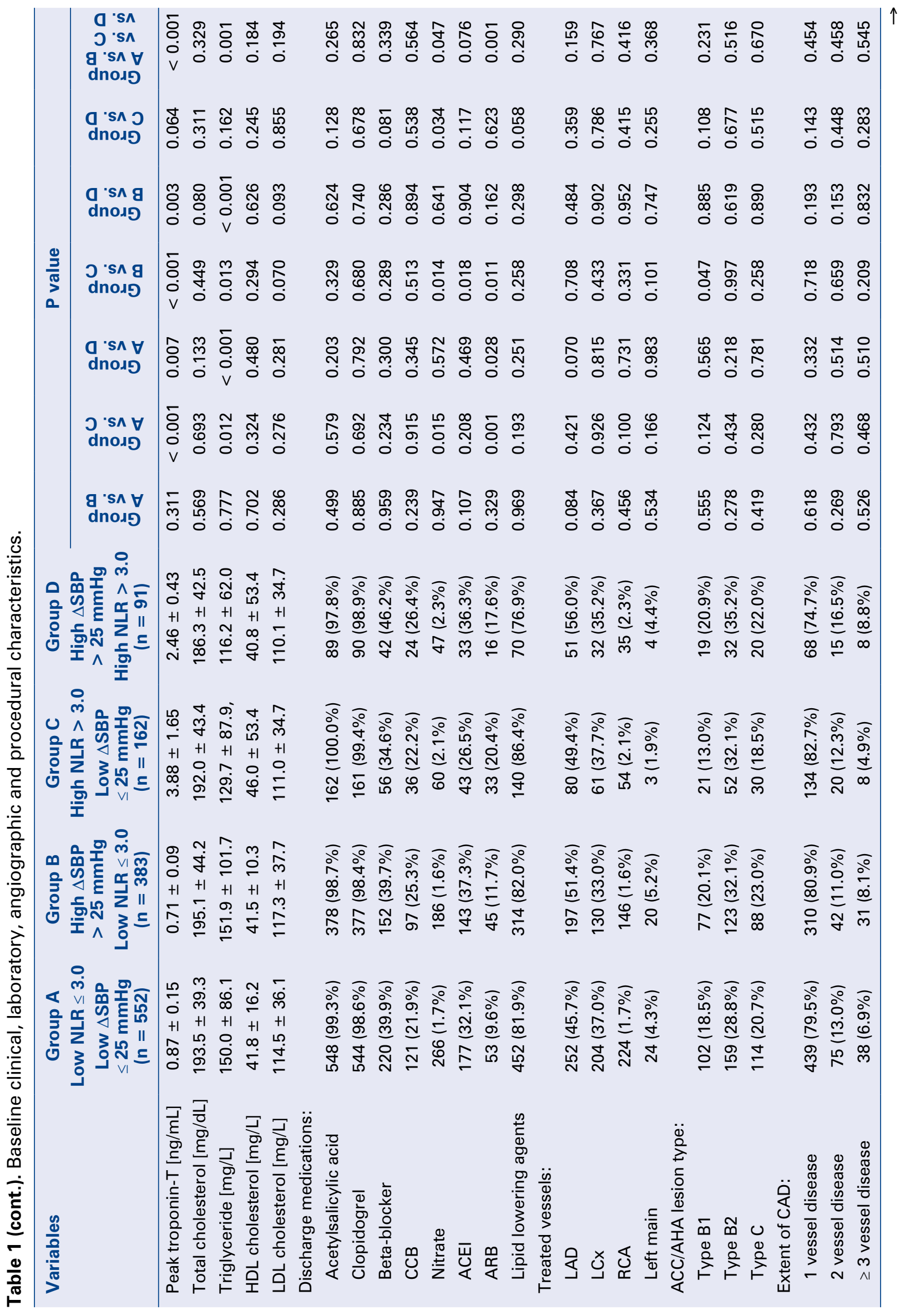




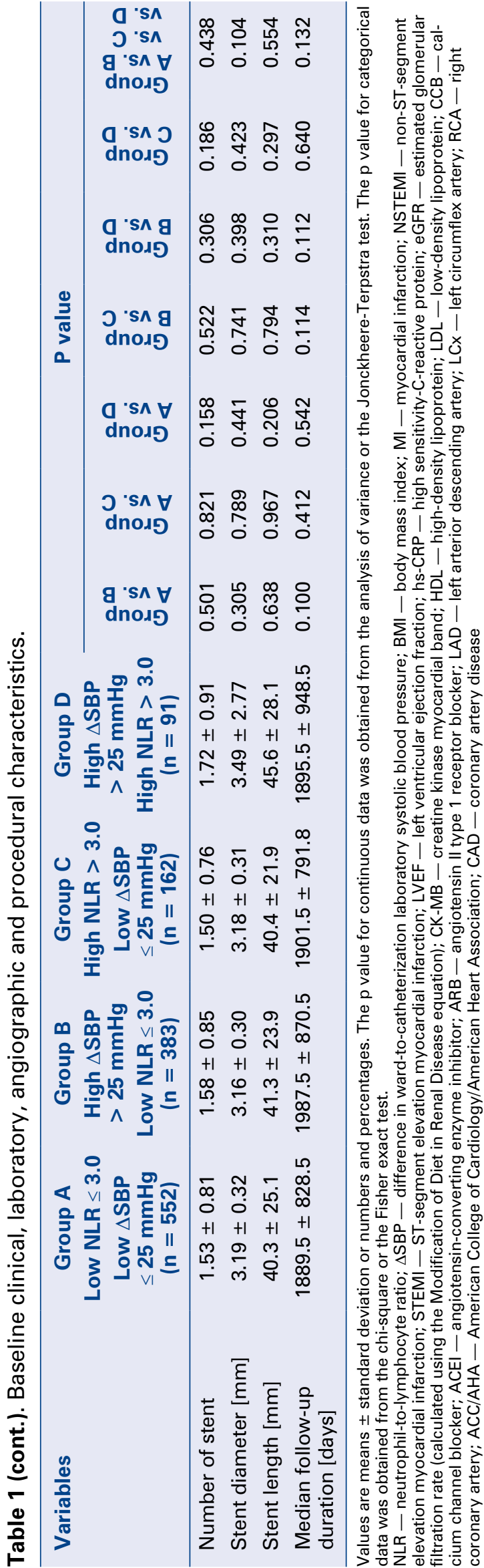

\section{Clinical outcomes}

The cumulative incidence of the primary endpoint, all-cause death, $\mathrm{CD}$, and secondary endpoint are summarized in Table 2, Figure 3, and Supplemental Online Material 1. After adjustment, the cumulative incidence of the primary endpoint in group D was significantly higher than that in group A (adjusted hazard ratio [aHR]: $1.920 ; 95 \%$ CI: $1.462-2.522 ; \mathrm{p}<0.001)$, group B (aHR: 1.751; 95\% CI: 1.186-2.584; $\mathrm{p}=0.005$ ), and group C (aHR: 3.514; 95\% CI: 1.481-9.640; $\mathrm{p}=0.015$ ) (Table 2, Fig. 3A). Similarly, the cumulative incidence of all-cause death was significantly higher in group D than in group A (aHR: 2.466; 95\% CI: 1.721-3.532; p < 0.001), group B (aHR: 1.767; 95\% CI: $1.168-2.603 ; \mathrm{p}=0.007$ ), and group C (aHR: 3.191; 95\% CI: 1.065-9.557; $p=0.038$ ) (Table 2, Fig. $3 \mathrm{~B})$. The cumulative incidence of $\mathrm{CD}$ in group $\mathrm{D}$ was significantly higher than that in group A (aHR: 3.394; 95\% CI: $1.627-7.079 ; \mathrm{p}=0.001)$ and group B (aHR: 3.185; 95\% CI: 1.228-7.014; $\mathrm{p}=0.017$ ) (Table 2, Fig. 3C). The cumulative incidence of secondary endpoint was not significantly different among the four groups (Suppl. Online Material 1, Fig. 3D). Table 3 summarizes improvements in C-statistics in predicting the primary endpoint when high NLR and high $\triangle \mathrm{SBP}$ were added into the model with conventional and meaningful clinical and procedural risk factors. The addition of high NLR and high $\triangle \mathrm{SBP}$ led to significant improvements in C-statistics from 0.575 to $0.602(\mathrm{p}=0.017)$ and $0.622(\mathrm{p}=0.004)$, respectively. However, the greatest improvement in C-statistics was seen when both high NLR and high $\triangle \mathrm{SBP}$ were combined into the model, with $\mathrm{C}$-statistics increasing significantly to 0.635 $(p=0.002)$. Table 4 shows independent predictors of the primary and secondary endpoints at 5 years. Low LVEF ( $<50 \%)$, hypertension, lymphocyte count, and catheterization laboratory SBP were found to be meaningful independent predictors of the primary endpoint. Additionally, diabetes mellitus was found to be a meaningful independent predictor of the secondary endpoint.

\section{Discussion}

The main findings of this study are the follows. The cumulative incidence of the primary endpoint in group D was significantly higher than that in the other three groups and the incorporation of high NLR and high $\triangle \mathrm{SBP}$ into the model with conventional and meaningful clinical and procedural risk factors synergistically increased the ability to predict the primary endpoint. 


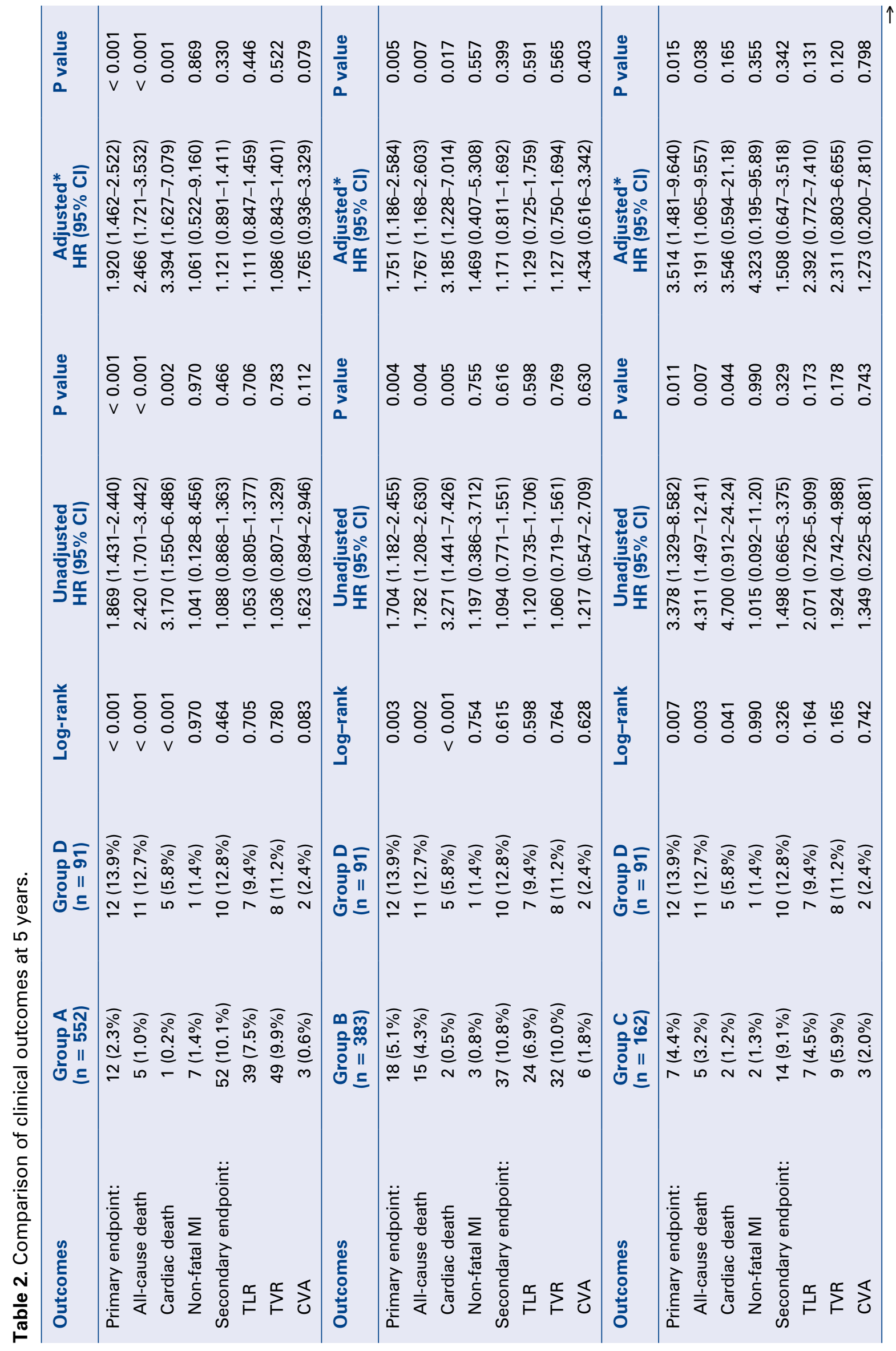




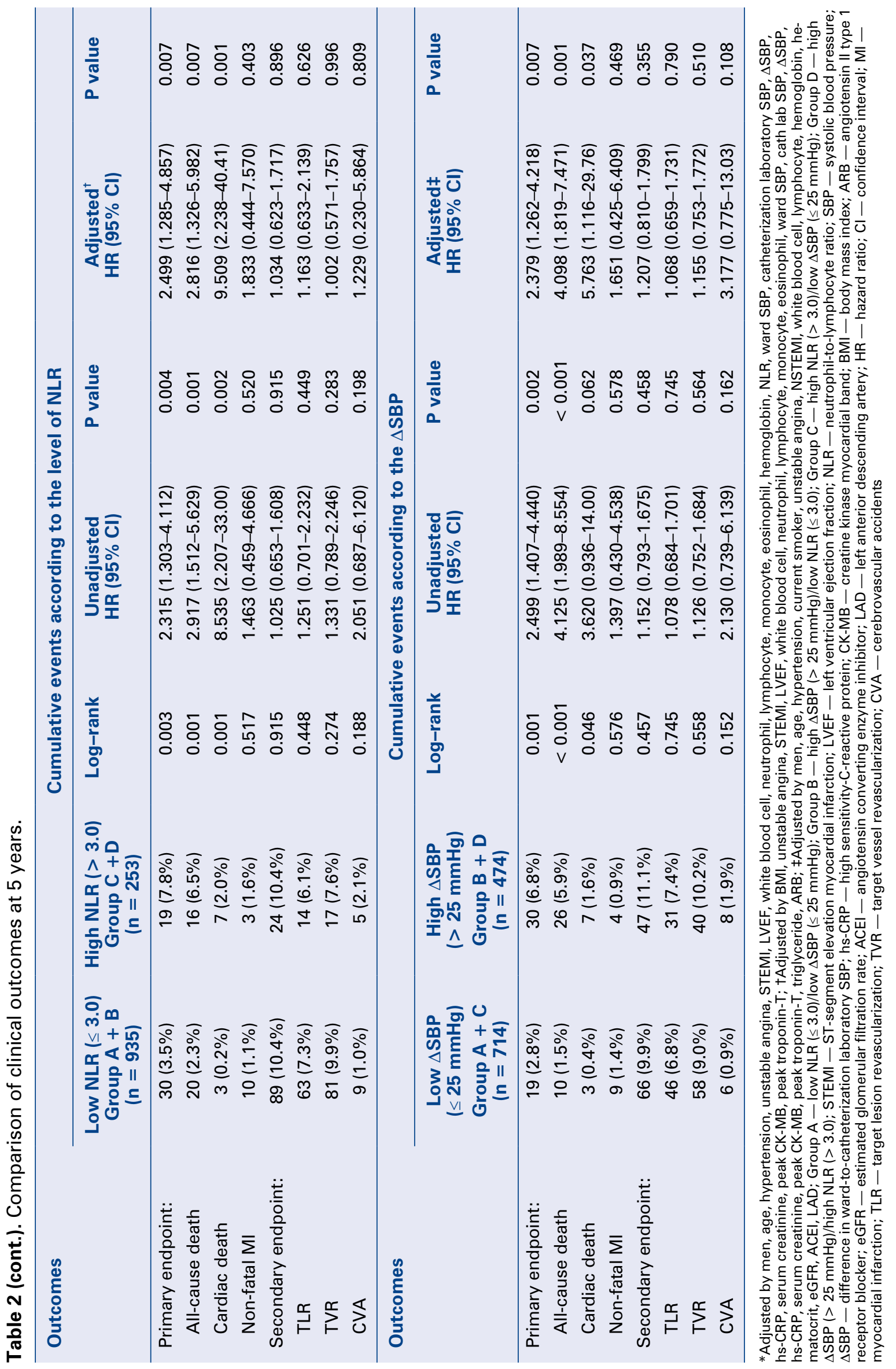




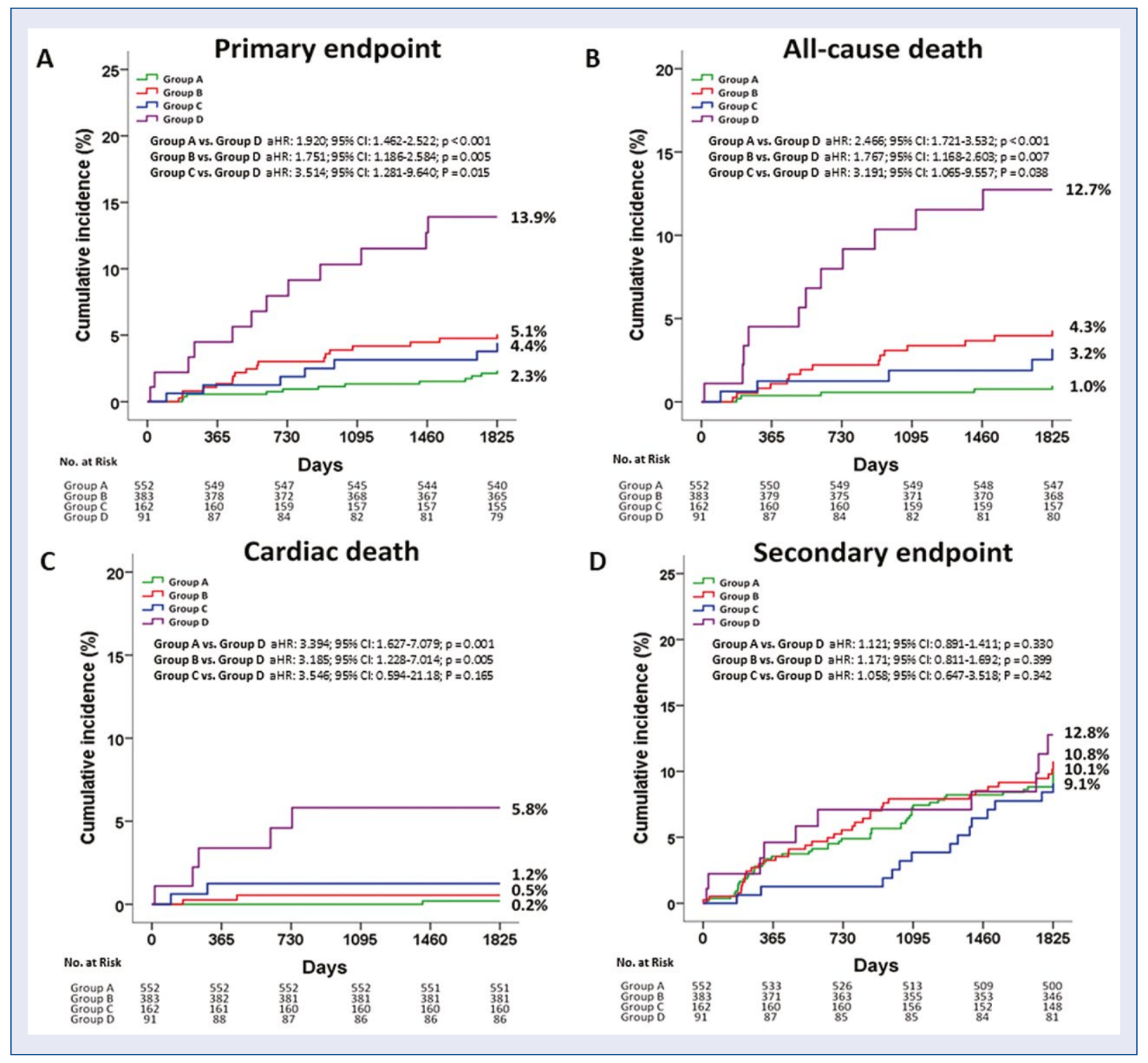

Figure 3. Kaplan-Meier analysis for primary outcome (A), all-cause death (B), cardiac death (C), and secondary end-point (D).

Table 3. C-statistics for Cox regression models for prediction of primary endpoint.

\begin{tabular}{|c|c|c|c|}
\hline Meaningful risk factors, NLR and $\triangle \mathrm{SBP}$ & C-statistics & $\begin{array}{l}\text { Estimated difference } \\
\qquad(95 \% \mathrm{Cl})\end{array}$ & $P$ value \\
\hline Meaningful clinical and procedural risk factors* & 0.575 & Reference & Reference \\
\hline $\begin{array}{l}\text { Meaningful clinical and procedural risk factors } \\
\text { plus high NLR }\end{array}$ & 0.602 & $0.055(-0.107$ to 0.108$)$ & 0.017 \\
\hline $\begin{array}{l}\text { Meaningful clinical and procedural risk factors } \\
\text { plus high } \triangle S B P\end{array}$ & 0.622 & $0.050(-0.099$ to 0.099$)$ & 0.004 \\
\hline $\begin{array}{l}\text { Meaningful clinical and procedural risk factors } \\
\text { plus high NLR and high } \Delta S B P\end{array}$ & 0.635 & $0.048(-0.093$ to 0.094$)$ & 0.002 \\
\hline
\end{tabular}

* Meaningful clinical and procedural risk factors composed of men, age, hypertension, unstable angina, non-STEMI, STEMI, left ventricular ejection fraction, white blood cell, neutrophil, lymphocyte, monocyte, eosinophil, hemoglobin, NLR, ward SBP, catheterization laboratory SBP, $\triangle \mathrm{SBP}$, hs-CRP, serum creatinine, peak CK-MB, peak troponin-T; NLR - neutrophil-to-lymphocyte ratio; SBP - systolic blood pressure; $\triangle \mathrm{SBP}$ - difference in ward-to-catheterization laboratory SBP; STEMI - ST-segment elevation myocardial infarction; hs-CRP — high sensitivity-C-

-reactive protein; $\mathrm{CK}-\mathrm{MB}$ — creatine kinase myocardial band; $\mathrm{Cl}$ - confidence interval 


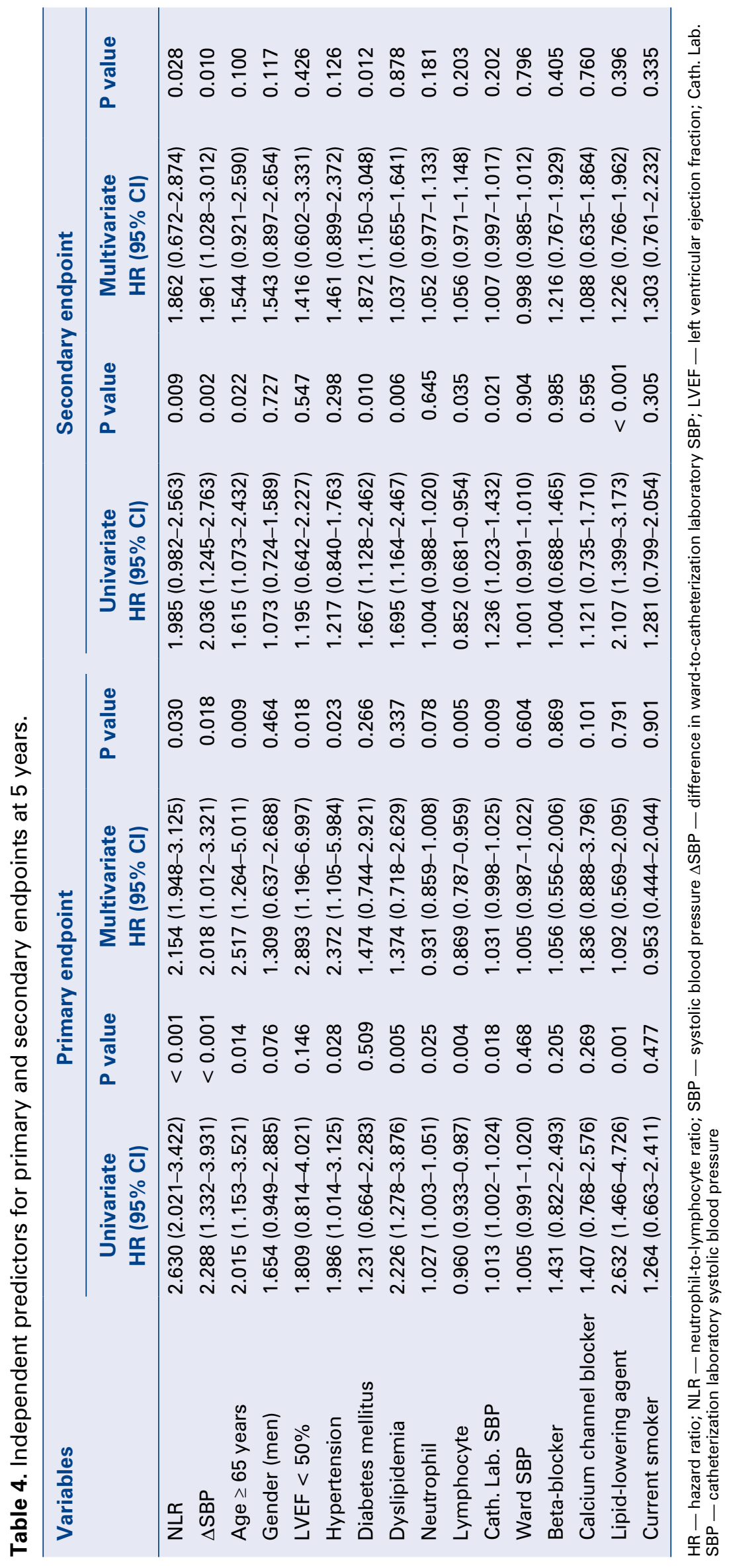


Because previous work has highlighted the importance of the NLR and $\triangle \mathrm{SBP}$ in predicting long-term major clinical outcomes in patients with ACS who underwent PCI, we investigated combined usefulness of these two non-invasive, inexpensive, relatively simple, not to mention the little time required employing the diagnostic tools in this study. The present study showed the additive value and combined usefulness of the NLR and $\triangle \mathrm{SBP}$ in predicting the primary endpoint in patients with ACS after DES implantation. According to available research, this study is the first to report the additive benefit of the NLR and $\triangle \mathrm{SBP}$ in predicting the 5-year follow-up of clinical outcomes in patients with ACS undergoing PCI with DES implantation.

Vascular inflammation plays a critical role in the initiation, evolution, and rupture of atherosclerotic plaques [22]. The circulating biomarkers of this process predict morbidity and mortality in patients with established CAD [23, 24]. To date, there has been diverse evidence regarding the role of the NLR, which suggests that it has an association with $\mathrm{CAD}[25,26]$ and that it could predict adverse in-hospital mortality and long-term mortality up to 3 years [26]. In this study, the NLR had an additional good prognostic value for predicting the primary endpoint during the 5-year follow-up (increase in C-statistics from 0.575 to $0.602 ; \mathrm{p}=$ $=0.017)$. In addition, the cumulative incidence of the primary endpoint was higher in the high NLR group than in the low NLR group (aHR: $2.499 ; 95 \%$ CI: $1.285-4.857 ; \mathrm{p}=0.007$; Table 2 ). These results are compatible with those of previous reports $[3,5$, 25]. In patients with ACS, a low lymphocyte count is common and can be explained by the elevated cortisol level, which induces apoptosis [27]. This low lymphocyte count (i.e., high NLR) is associated with adverse clinical outcomes [6]. In this study, the frequency of the primary endpoint was significantly higher in the high $\triangle \mathrm{SBP}$ group than in the low $\triangle$ SBP group at 5 years (aHR: $2.379 ; 95 \%$ CI: $1.262-4.218 ; \mathrm{p}=0.007$; Table 2). It is well known that target organ damage in patients with hypertension and cardiovascular complications is related to elevated $\mathrm{BP}$, which is determined from the average of multiple $\mathrm{BP}$ readings (mean $\mathrm{BP}$ ) [28]. $\triangle \mathrm{SBP}$ may not be a good substitute of 24 -hour ambulatory BP monitoring (ABPM). However, $\mathrm{ABPM}$ is an additional diagnostic test with inherent costs and takes more time to get results compared to $\triangle \mathrm{SBP}$. Therefore, we thought that $\triangle \mathrm{SBP}$ may be the preferred technique for the patients with ACS. Moreover, BP measurement in the ward and before catheterization is already done in routine clinical practice.

Although the NLR and $\triangle$ SBP have a different pathophysiological mechanism, they both lead to accelerated atherosclerosis. In patients with ACS, an increased total leukocyte count predicts mortality and recurrence of MI [29,30]. ACS is most commonly caused by disruption of atherosclerotic plaques with superimposed thrombus formation; thus, inflammation plays a crucial role in the pathogenesis of acute coronary events [31]. To date, the precise mechanism of acute transient BP elevation is less well known. Under stressful situations (e.g., CAG or PCI), increased activity of the hypothalamic-pituitary-adrenal axis culminating in release of with catecholamine play a vital role in initiating acute deviations from normal physiology such as hypertension. Through changes in the circulating catecholamine levels, emotional or psychosocial stress evokes negative effects on autonomic and hormonal homeostasis, which can lead to inflammation, metabolic abnormalities, endothelial dysfunction, hypertension, and insulin resistance [32]. From a different perspective, increased cardiac output and arterial stiffness under stressful circumstances are related to acute BP responses; the latter are associated with atherosclerosis [33, 34]. Her et al. [10] proposed that an acute elevation of BP under stress might contribute to an increased risk of adverse clinical outcomes, with this BP elevation representing significant arterial atherosclerosis and increased arterial stiffness. Another possible interaction between BP and NLR is that an elevated BP increases the formation of hydrogen peroxide and free radicals in the plasma [35], and these substrates cause decreased production of nitric oxide from the endothelium [36] and increased leukocyte adhesion [37]. Therefore, these two parameters are associated with each other. Because atherosclerosis is an inflammatory disease and an important causative factor of $\mathrm{BP}$ variations, early detection and modification of these reversible factors may reduce the frequency and severity of adverse cardiac events during longterm follow-up after implantation of DESs. In this regard, the combined use of the NLR and $\triangle \mathrm{SBP}$ as a predictive tool for adverse cardiac events is a rational approach, especially in patients undergoing PCI. Finally, although in this study shows the additional combined usefulness of the NLR and $\triangle \mathrm{SBP}$ in predicting long-term outcomes after implantation of DESs, other laboratory (e.g., GFR, hemoglobin), clinical features, or well-established risk assessment tools (e.g., Global Registries of 
Acute Coronary Events [GRACE] or TIMI risk scores) could also be used as alternatives for these two parameters in further studies.

\section{Limitations of the study}

This study has some limitations. First, the NLR was checked only once; thus, it was not known whether there was any change in its value. Second, even though there was an attempt to perform a multivariable Cox proportional hazard regression analysis including baseline confounding factors, as this study was a non-randomized retrospective single-center study with several exclusion criteria, selection bias cannot be excluded. Third, the sample size may not be sufficiently large enough to accurately estimate the study results. Larger randomized prospective studies are required to confirm these results. Fourth, group D was composed of a stringently selected population. Therefore, this may have led to selection bias. Fifth, because 24-hour ABPM was not performed in this study, assessment of any BP variability was not possible. Finally, the patients in this study were enrolled between September 2002 and August 2007; this limited study period can be considered to be the main limitation.

\section{Conclusions}

In conclusion, in this retrospective observational study of the patients with ACS, the incorporation of both high NLR and high $\triangle \mathrm{SBP}$ into the model with conventional and meaningful clinical and procedural risk factors increased the ability to predict the primary endpoint during the 5-year follow-up period.

\section{Conflict of interest: None declared}

\section{References}

1. Geovanini GR, Libby P. Atherosclerosis and inflammation: overview and updates. Clin Sci (Lond). 2018; 132(12): 1243-1252, doi: 10.1042/CS20180306, indexed in Pubmed: 29930142.

2. Koelwyn GJ, Corr EM, Erbay E, et al. Regulation of macrophage immunometabolism in atherosclerosis. Nat Immunol. 2018; 19(6): 526-537, doi: 10.1038/s41590-018-0113-3, indexed in Pubmed: 29777212.

3. Arbel Y, Shacham Y, Ziv-Baran T, et al. Higher neutrophil/lymphocyte ratio is related to lower ejection fraction and higher long-term all-cause mortality in ST-elevation myocardial infarction patients. Can J Cardiol. 2014; 30(10): 1177-1182, doi: 10.1016/j.cjca.2014.05.010, indexed in Pubmed: 25154806.

4. Arbel Y, Finkelstein A, Halkin A, et al. Neutrophil/lymphocyte ratio is related to the severity of coronary artery disease and clinical outcome in patients undergoing angiography. Athero- sclerosis. 2012; 225(2): 456-460, doi: 10.1016/j.atherosclerosis.2012.09.009, indexed in Pubmed: 23040448.

5. Xu Na, Tang XF, Yao Yi, et al. Predictive value of neutrophil to lymphocyte ratio in long-term outcomes of left main and/or three-vessel disease in patients with acute myocardial infarction. Catheter Cardiovasc Interv. 2018; 91(S1): 551-557, doi: 10.1002/ ccd.27495, indexed in Pubmed: 29330938.

6. Dentali F, Nigro O, Squizzato A, et al. Impact of neutrophils to lymphocytes ratio on major clinical outcomes in patients with acute coronary syndromes: a systematic review and meta-analysis of the literature. Int J Cardiol. 2018; 266: 31-37, doi: 10.1016/j.ijcard.2018.02.116, indexed in Pubmed: 29887466.

7. Kivimäki M, Steptoe A. Effects of stress on the development and progression of cardiovascular disease. Nat Rev Cardiol. 2018; 15(4): 215-229, doi: 10.1038/nrcardio.2017.189, indexed in Pubmed: 29213140 .

8. Hamer M. Psychosocial stress and cardiovascular disease risk: the role of physical activity. Psychosom Med. 2012; 74(9): 896-903, doi: 10.1097/PSY.0b013e31827457f4, indexed in Pubmed: 23107839.

9. Voigt C, Münch J, Avanesov M, et al. Early segmental relaxation abnormalities in hypertrophic cardiomyopathy for differential diagnostic of patients with left ventricular hypertrophy. Clin Cardiol. 2017; 40(11): 1026-1032, doi: 10.1002/clc.22761, indexed in Pubmed: 28741295.

10. Her AY, Ann SH, Lee JH, et al. Differences in ward-to-cath lab systolic blood pressure predicts long-term adverse outcomes after drug-eluting stent implantation. Heart Vessels. 2015; 30(6): 740-745, doi: 10.1007/s00380-014-0550-3, indexed in Pubmed: 25062712.

11. Hochheiser LI, Juusola JL, Monane M, et al. Economic utility of a blood-based genomic test for the assessment of patients with symptoms suggestive of obstructive coronary artery disease. Popul Health Manag. 2014; 17(5): 287-296, doi: 10.1089/ pop.2013.0096, indexed in Pubmed: 24568603.

12. Chida Y, Steptoe A. Greater cardiovascular responses to laboratory mental stress are associated with poor subsequent cardiovascular risk status: a meta-analysis of prospective evidence. Hypertension. 2010; 55(4): 1026-1032, doi: 10.1161/HYPERTENSIONAHA.109.146621, indexed in Pubmed: 20194301.

13. Grech $\mathrm{ED}$. ABC of interventional cardiology: percutaneous coronary intervention. II: the procedure. BMJ. 2003; 326(7399): 1137-1140, doi: 10.1136/bmj.326.7399.1137, indexed in Pubmed: 12763994.

14. Kim YH, Her AY, Jeong MHo, et al. A comparison between statin with ACE inhibitor or ARB therapy in STEMI patients who underwent successful PCI with drug-eluting stents. Atherosclerosis. 2019; 289: 109-117, doi: 10.1016/j.atherosclerosis.2019.08.018, indexed in Pubmed: 31491742.

15. Hong SJ, Kim BK, Shin DH, et al. IVUS-XPL Investigators. Effect of intravascular ultrasound-guided vs angiography-guided everolimus-eluting stent implantation: the IVUS-XPL randomized clinical trial. JAMA. 2015; 314(20): 2155-2163, doi: 10.1001/jama.2015.15454, indexed in Pubmed: 26556051.

16. O'Gara PT, Kushner FG, Ascheim DD, et al. 2013 ACCF/AHA guideline for the management of ST-elevation myocardial infarction: a report of the American College of Cardiology Foundation/American Heart Association Task Force on Practice Guidelines. Circulation. 2013; 127(4): e362-e425, doi: 10.1161/ CIR.0b013e3182742cf6, indexed in Pubmed: 23247304. 
17. Kim YH, Her AY, Jeong MHo, et al. Impact of renin-angiotensin system inhibitors on long-term clinical outcomes in patients with acute myocardial infarction treated with successful percutaneous coronary intervention with drug-eluting stents: Comparison between STEMI and NSTEMI. Atherosclerosis. 2019; 280: 166-173, doi: 10.1016/j.atherosclerosis.2018.11.030, indexed in Pubmed: 30529829.

18. Matsushita K, Mahmoodi BK, Woodward M, et al. Comparison of risk prediction using the CKD-EPI equation and the MDRD study equation for estimated glomerular filtration rate. JAMA. 2012; 307(18): 1941-1951, doi: 10.1001/jama.2012.3954, indexed in Pubmed: 22570462.

19. Kim YH, Her AY, Jeong MHo, et al. Impact of stent generation on 2-year clinical outcomes in ST-segment elevation myocardial infarction patients with multivessel disease who underwent culprit-only or multivessel percutaneous coronary intervention. Catheter Cardiovasc Interv. 2020; 95(2): E40-E55, doi: 10.1002/ ccd.28440, indexed in Pubmed: 31423723.

20. Pencina MJ, D'Agostino RB. Overall C as a measure of discrimination in survival analysis: model specific population value and confidence interval estimation. Stat Med. 2004; 23(13): 2109-2123, doi: 10.1002/sim.1802, indexed in Pubmed: 15211606.

21. Efron B, Tibshirani R. An Introduction to the Bootstrap. New York, NY: Chapman \& Hall. 1993, doi: 10.1007/978-1-4899-4541-9.

22. Ross R. Atherosclerosis: an inflammatory disease. N Engl J Med. 1999; 340(2): 115-126, doi: 10.1056/NEJM199901143400207, indexed in Pubmed: 9887164.

23. Morrow D, Rifai N, Antman E, et al. C-Reactive protein is a potent predictor of mortality independently of and in combination with troponin $\mathrm{t}$ in acute coronary syndromes: a TIMI $11 \mathrm{~A}$ substudy. J Am Coll Cardiol. 1998; 31(7): 1460-1465, doi: 10.1016/ s0735-1097(98)00136-3.

24. Lindahl B, Toss H, Siegbahn A, et al. Markers of myocardial damage and inflammation in relation to long-term mortality in unstable coronary artery disease. FRISC Study Group. Fragmin during Instability in Coronary Artery Disease. N Engl J Med. 2000; 343(16): 1139-1147, doi: 10.1056/NEJM200010193431602, indexed in Pubmed: 11036119.

25. Kaya MG, Akpek M, Lam YY, et al. Prognostic value of neutrophil/lymphocyte ratio in patients with ST-elevated myocardial infarction undergoing primary coronary intervention: a prospective, multicenter study. Int J Cardiol. 2013; 168(2): 1154-1159, doi: 10.1016/j.ijcard.2012.11.074, indexed in Pubmed: 23219132.

26. Sen N, Afsar B, Ozcan F, et al. The neutrophil to lymphocyte ratio was associated with impaired myocardial perfusion and long term adverse outcome in patients with ST-elevated myocardial infarction undergoing primary coronary intervention. Atherosclerosis. 2013; 228(1): 203-210, doi: 10.1016/j.atherosclerosis.2013.02.017, indexed in Pubmed: 23489347.
27. Nelson DH, Sandberg AA, Palmer JG, et al. Blood levels of 17-hydroxycorticosteroids following the administration of adrenal steroids and their relation to levels of circulating leukocytes. J Clin Invest. 1952; 31(9): 843-849, doi: 10.1172/JCI102671, indexed in Pubmed: 12981169.

28. Mancia G, Fagard R, Narkiewicz K, et al. 2013 ESH/ESC Guidelines for the management of arterial hypertension: the Task Force for the management of arterial hypertension of the European Society of Hypertension (ESH) and of the European Society of Cardiology (ESC). J Hypertens. 2013; 31(7): 1281-1357, doi: 10.1097/01.hjh.0000431740.32696.cc, indexed in Pubmed: 23817082.

29. Sabatine M, Morrow D, Cannon C, et al. Relationship between baseline white blood cell count and degree of coronary artery disease and mortality in patients with acute coronary syndromes. J Am Coll Cardiol. 2002; 40(10): 1761-1768, doi: 10.1016/s07351097(02)02484-1.

30. Zhang S, Diao J, Qi C, et al. Predictive value of neutrophil to lymphocyte ratio in patients with acute ST segment elevation myocardial infarction after percutaneous coronary intervention: a meta-analysis. BMC Cardiovasc Disord. 2018; 18(1): 75, doi: 10.1186/s12872-018-0812-6, indexed in Pubmed: 29716535.

31. Sakakura K, Nakano M, Otsuka F, et al. Pathophysiology of atherosclerosis plaque progression. Heart Lung Circ. 2013; 22(6): 399-411, doi: 10.1016/j.hlc.2013.03.001, indexed in Pubmed: 23541627.

32. Curtis BM, O'Keefe JH. Autonomic tone as a cardiovascular risk factor: the dangers of chronic fight or flight. Mayo Clin Proc. 2002; 77(1): 45-54, doi: 10.4065/77.1.45, indexed in Pubmed: 11794458.

33. Jones A, Steeden JA, Pruessner JC, et al. Detailed assessment of the hemodynamic response to psychosocial stress using realtime MRI. J Magn Reson Imaging. 2011; 33(2): 448-454, doi: 10.1002/jmri.22438, indexed in Pubmed: 21274988.

34. Sunbul M, Seckin D, Durmus E, et al. Assessment of arterial stiffness and cardiovascular hemodynamics by oscillometric method in psoriasis patients with normal cardiac functions. Heart Vessels. 2015; 30(3): 347-354, doi: 10.1007/s00380-014-0490-y, indexed in Pubmed: 24633494.

35. Lacy F, O'Connor DT, Schmid-Schönbein GW. Plasma hydrogen peroxide production in hypertensives and normotensive subjects at genetic risk of hypertension. J Hypertens. 1998; 16(3): 291-303, doi: 10.1097/00004872-199816030-00006, indexed in Pubmed: 9557922.

36. Vanhoutte PM, Boulanger CM. Endothelium-dependent responses in hypertension. Hypertens Res. 1995; 18(2): 87-98, doi: 10.1291/hypres.18.87, indexed in Pubmed: 7584924.

37. Swei A, Lacy F, DeLano FA, et al. Oxidative stress in the Dahl hypertensive rat. Hypertension. 1997; 30(6): 1628-1633, doi: 10.1161/01.hyp.30.6.1628, indexed in Pubmed: 9403593. 